\title{
Versão Portuguesa Reduzida da Escala de Avaliação da Aparência de Derriford (DAS-14): Análise fatorial exploratória e confirmatória
}

\section{Derriford Appearance Scale (DAS-14) short Portuguese version: Exploratory and confirmatory analyse}

Artigo Original | Original Article

\section{José Mendes PhD (1a), Vera Pereira Estudante PhD $(2,3 b)$}

(1) INTELECTO - Psicologia e Investigação, Ponta Delgada, Açores, Portugal

(2) FCHS-UALG - Faculdade de Ciências Humanas e Sociais, Universidade do Algarve, Campus de Gambelas, Faro, Portugal

(3) $\mathrm{CIEO}$ - Reserch Center for Spatial and Organizational Dynamics, Faro, Portugal

(a) Recolha de Dados, Revisão de Literatura, Análise Estatística, Interpretação e Discussão de Resultados, Redação do Manuscrito.

(b) Recolha de dados, Revisão de Literatura, Revisão do Manuscrito.

Autor para correspondência | Corresponding author: José Carlos da Silva Mendes; Rua do Monte, 52B, Fração L, R/C Esquerdo Nascente, Ilha de São Miguel, Açores; josemendes@intelecto.pt

\section{Palavras-Chave}

Autoconsciência da aparência

Escala de avaliação

DAS-14

\section{Keywords}

Confirmatory analysis

Body image

Assessment measures

Muscularity

Body fat

Men

\section{RESUMO}

Objetivo: Pretende-se com este estudo, apresentar uma versão reduzida da Escala de Avaliação da Aparência de Derriford (DAS-24) para a população portuguesa.

Método: Após análise de seis amostras recolhidas entre os anos 2010 e 2017, num total de 1016 participantes que responderam a questões relacionadas com o investimento esquemático da aparência e autoconsciência da aparência (DAS-24), solicitou-se autorização aos autores da versão portuguesa e versão original da DAS-24 a redução da escala para 14 itens.

Resultados: A DAS-14 apresentou um bom índice de consistência interna, quer na amostra não-clínica ( $\alpha$ de Cronbach $=0,91)$, quer na amostra clínica ( $\alpha$ de Cronbach $=0,88$ ). A análise fatorial confirmatória apresentou um ajustamento aceitável, quer para a amostra não clínica $\left(\chi^{2} / g l=1,17 ; G F I=0,95 ; C F I=0,99 ; T L I=0,98\right.$; $R M S E A=0,028 ; p[(R M S E A \leq 0,05)=0,92]$, quer para a amostra clínica $\left(\chi^{2} / g l=1,36 ; G F I=0,94 ; C F I=0,98\right.$; $T L I=0,96 ;$ RMSEA $=0,047 ; p[($ RMSEA $\leq 0,05)=0,56]$.

Conclusões: A DAS-14 apresenta-se psicometricamente robusta na avaliação da autoconsciência da aparência em amostras da população geral e clínica.

\section{ABSTRACT}

Aim: The present study intends to evaluate the factorial validity and reliability of a reduced version of the Derriford Appearance Scale (DAS-14) for the Portuguese population.

Method: After analysis of six samples collected between 2010 and 2017, a total of 1016 participant answered questions related to the schematic investment of appearance and self-consciousness of appearance (DAS-24), authorization was requested to the authors of the original and Portuguese version of the DAS-24 to reduce the scale to 14 items.

Results: The DAS-14 presented good internal consistency to non-clinical sample (Cronbach's $\alpha=0.91$ ) and (Cronbach's $\alpha=0.88$ ) to clinical samples. The Confirmatory Factor Analysis of the DAS-14, presents an acceptable to non-clinical sample $\left(\chi^{2} / g l=1.17 ; G F I=0.95 ; C F I=0.99 ; T L I=0.98 ; R M S E A=0.028 ; p[r m s e a\right.$ $\leq .05]=0.92)$, and clinical sample $\left(\chi^{2} / g I=1.36 ; G F I=0.94 ; C F I=0.98 ; T L I=0.96 ; R M S E A=0.047 ; p[\mathrm{rmsea}\right.$ $\leq .05]=0.56$ ).

Conclusion: The Portuguese version of the DAS-14 is psychometrically robust in evaluating self-consciousness of appearance in general and clinical population. 


\section{INTRODUÇÃO}

As preocupações com a aparência são um elemento central nas experiências e interações sociais, conduzindo à construção da identidade de um indivíduo, em que a aparência comunica informação relativa à etnicidade, ao caráter e ao estado físico e emocional (Carr, Harris, \& James, 2000). Níveis elevados de preocupação com a aparência influenciam a que os indivíduos tenham uma perceção mais negativa do seu meio ambiente (Rosser, Moss, \& Rumsey, 2010).

Na última década, a preocupação com a aparência em Portugal tem despertado interesse na comunidade científica, tendo sido realizados vários estudos: revisões de literatura (Mendes \& Figueiras, 2013; Mendes, Rego, Figueiras, \& Moss, 2018); estudos empíricos em amostras não-clínicas (Mendes, Figueiras, \& Moss, 2016) e amostras clínicas (Alves, 2016; Mendes, Rego, Figueiras, Moss, \& Alcaidinho, 2018); tradução e validação de escalas que avaliam o investimento esquemático da aparência (Nazaré, Moreira, \& Canavarro, 2010) e avaliação da autoconsciência da aparência (Mendes, Figueiras, Moreira, \& Moss, 2016).

A construção da Derriford Appearance Scale (DAS-59) contribui para ultrapassar/ veio ultrapassar as limitações de vários instrumentos de avaliação do estado de saúde mental, personalidade ou qualidade de vida em relação à imagem corporal. O objetivo desta escala é avaliar as dificuldades experienciadas do indivíduo em viver com problemas relativamente à sua aparência (Carr et al., 2000). Através de 59 itens distribuídos por um fator geral (autoconsciência da aparência) e quatro dimensões (autoconsciência da aparência em situações sociais, autoconsciência das características sexualmente significativas do corpo, baixa autoestima e autoconsciência das características faciais), a Derriford Appearance Scale apresenta-se válida e confiável, na avaliação do sofrimento e dificuldades em viver com problemas de aparência (Carr et al., 2000; Harris \& Carr, 2001b). A DAS-59 apresentou-se como uma medida objetiva do espectro de angústia psicológica e disfunção, que é característica do desfiguramento, deformidades e problemas estéticos da aparência (Harris \& Carr, 2001a).

Face à extensão da respetiva escala, em 2005, Carr, Moss e Harris, apresentaram a versão reduzida da Derriford Appearance Scale em 24 itens, com propriedades psicométricas robustas e identidade concetual da versão longa. A DAS-24 apresenta-se como uma escala unidimensional que avalia a autoconsciência da aparência, quer em populações não-clínicas, quer em populações clínicas (Mendes \& Figueiras, 2013). No entanto, estudos que envolvam vários questionários tornam-se extensos, não sendo adequados para pessoas fragilizadas ou acamadas (Ribeiro, 2010), apresentando elevada probabilidade de não serem respondidos (Chaer, Diniz, \& Ribeiro, 2011). O elevado número de itens pode influenciar a compreensão das questões (Leeuw, Hox, \& Dillman, 2008) e tender à existência do aumento do número de questões por responder (Stanton, Sinar, Balzer, \& Smith, 2002).

Considerando que as versões reduzidas de escalas têm sido consideradas um assunto sensível devido à informação recolhida ser restrita e às possíveis qualidades psicométricas serem duvidosas (Ziegler, Kemper, \& Kruyen, 2014), pretende-se com este trabalho apresentar uma versão reduzida da DAS-24, tendo em consideração uma seleção de itens sem que se coloquem em causa as características métricas da estrutura original.

Segundo Stanton et al. (2002), a redução de um instrumento deve ter em consideração três importantes etapa: uma breve revisão de literatura, a demonstração dos procedimentos da redução da escala e, por último, a aplicação das melhores práticas e compromissos viáveis na seleção dos itens. Assim, através de uma revisão de literatura sobre os critérios a considerar na escolha dos itens, respeito pelas especificadas linguísticas e uma uniformização da versão reduzida com a versão original, pretende-se com este estudo, apresentar uma versão reduzida da DAS-24, contribuindo para uma melhor aplicabilidade de escalas de avaliação das preocupações com a aparência em contexto clínico.

\section{MÉTODO}

A pertinência de desenvolver uma escala reduzida das DAS-24, principalmente em contexto clínico, levou a uma breve análise de várias amostras recolhidas nos últimos 10 anos, tendo-se proposto a redução da DAS-24 aos autores originais e autores da versão portuguesa. Após análise prévia das respetivas amostras por parte dos autores originais e da versão portuguesa, obteve-se autorização para a redução da DAS-24.

\section{Procedimentos e Participantes}

A amostra global do presente estudo ficou constituída por um total de 1016 participantes, tendo sido usado 4 amostras não-clínicas e 2 amostras clínicas recolhidas entre o ano 2010 e o ano 2017. As seis amostras destinaram-se a vários estudos académicos, incluindo duas teses de doutoramento finalizadas, validação da DAS-24 e outros trabalhos que aguardam publicação. Do total de participantes, 427 (42\%) eram homens e 589 (58\%) mulheres, com idades compreendidas entre os $18 \mathrm{e}$ os 78 anos $(M=35,76 ; D P=12,60)$. 
A amostra total dividiu-se numa amostra clínica (i.e., participantes que apresentaram uma diferença visível na face) e não-clínica (i.e., participantes sem diferenças visíveis na face). A amostra clínica englobou 171 participantes com idades compreendidas entre os 21 e os 70 anos $(M=40,77$ anos; $D P=10,78)$ e maioritariamente do sexo masculino $(n$ $=109 ; 63,7 \%)$. A amostra não-clínica englobou 845 participantes com idades compreendida entre os 18 e os 78 anos $(M=34,75$ anos; $D P=12,70)$, maioritariamente do sexo feminino $(n=527 ; 62,4 \%)$. A recolha da amostra clínica teve a aprovação da comissão de ética do Centro Hospitalar Lisboa Norte, EPE (com a referência PCA-25 JUN 20120454). Todos os participantes que responderam ao questionário em formato papel assinaram o termo de consentimento informado e esclarecido.

A amostra-não clínica foi recolhida através de questionários on-line e por conveniência, tendo como critério de inclusão, idade igual ou superior a 18 anos e a amostra clínica foi recolhida na unidade de internamento da cirurgia plástica e reconstrutiva do Hospital de Santa Maria, tendo como critério de inclusão uma diferença visível na face (cicatriz, queimadura, mancha, outros) e idade igual ou superior a 18 anos.

\section{Instrumentos}

DAS-24. A versão portuguesa da Derriford Appearance Scale - short (Carr et al., 2005; Mendes et al., 2016) é um instrumento que se inicia com uma questão sobre a existência de algum aspeto da aparência que preocupa o indivíduo e três questões abertas. Seguidamente surgem 24 itens de tipo Likert, que pretendem avaliar a autoconsciência da aparência (forma como o indivíduo se sente e comporta relativamente ao aspeto que o incomoda), sendo cotados através das seguintes opções de resposta: $1=\mathrm{Nada}, 2=$ Ligeiramente, 3 = Moderadamente e $4=$ Extremamente ou de $0=\mathrm{N} / \mathrm{A}$ (não aplicável), ou $1=$ Nunca/Quase nunca, $2=$ Às vezes, $3=$ Frequentemente e $4=$ Quase Sempre. Por último, outras duas questões adicionais pretendem avaliar até que ponto o aspeto da aparência do indivíduo causa dor/desconforto ou o limita na capacidade física no seu quotidiano. A escala apresenta um bom índice de consistência interna ( $\alpha$ de Cronbach $=0,91$ ).

ASI-R. A versão portuguesa da Appearance Schemas Inventory - revista (Cash, Melnyk, \& Hrabosky, 2004; Nazaré et al., 2010) é composta pelos fatores Saliência Autoavaliativa e Saliência Motivacional, avaliando o investimento esquemático na aparência através de 20 itens de autorresposta, numa escala tipo Likert com uma cotação que varia entre 1 (Discordo fortemente) e 5 (Concordo fortemente). Este inventário avalia o investimento da imagem corporal e os esforços de um indivíduo em manter ou aumentar a sua atratividade física e gerir a aparência, apresentando bom índice de consistência interna ( $\alpha$ de Cronbach $=0,89$ ).

\section{Análise Estatística}

Recorreu-se ao software SPSS24 para o sistema operativo MacOS e AMOS21 para sistema operativo Windows, para a realização do tratamento estatístico dos dados. Procedeu-se a uma análise fatorial exploratória (AFE) das seis amostras, verificaram-se os alfa $(\alpha)$ de Cronbach de forma a estimar a fiabilidade do instrumento (Marôco \& Garcia-Marques, 2006).

Utilizou-se uma regressão linear múltipla para analisar a versão reduzida dos itens. A pontuação total da versão portuguesa original da DAS-24 foi utilizada como variável dependente e cada item como variável independente. Posteriormente, analisou-se o coeficiente de correlação de Pearson ( $r$ ) para quantificar a intensidade e a direção da associação entre as variáveis dos instrumentos DAS-14 (versão reduzida da DAS-24) e ASI-R.

Procedeu-se à análise fatorial confirmatória (AFC), com a estimação de máxima verosimilhança, avaliando a qualidade de ajustamento global do modelo fatorial pelo teste Qui-quadrado de Ajustamento $\left(\chi^{2}\right)$, considerando $\chi^{2} / g /$ inferior a 5 como modelo aceitável; de acordo com os índices Comparative Fit Index (CFI), Goodness of Fit Index (GFI), Root Mean Square Error of Approximation (RMSEA), Incremental Fit Index (IFI), sendo considerado um bom ajustamento quando os índices são próximos do valor de 1 e o RMSEA apresenta valores de referência inferiores a 0,05 (Marôco, 2014).

A fiabilidade compósita ( $\mathrm{FC}$ ) e a variância extraída média (VEM) foram avaliadas através das fórmulas (Marôco, 2014): $\widehat{F C}=\frac{\left(\sum_{i t=1}^{24} \lambda\right)^{2}}{\left(\sum_{i t=1}^{24} \lambda\right)^{2}+\sum_{i t=1}^{24} \varepsilon}$ e $\widehat{V E M}=\frac{\sum_{i t=1}^{24} \lambda^{2}}{\sum_{i t=1}^{24} \lambda^{2}+\sum_{i t=1}^{24} \varepsilon}$.

\section{RESULTADOS}

\section{Desenvolvimento da Versão Reduzida da DAS-24}

Os itens foram analisados individualmente com suporte numa revisão de literatura de estudos realizados em Portugal sobre questões relacionadas com a aparência. Posteriormente, procedeu-se a uma análise fatorial exploratória em cada uma das seis amostras, de forma a analisar os itens com baixas correlações Item-Total corrigido. Por fim, prosseguiu-se com o pedido de autorização aos autores da versão portuguesa e versão original da DAS-24, na redução dos itens. Posteriormente, reuniram-se os dados em duas amostras (clínica e nãoclínica), analisando-se as correlações Item-Total e eliminando os itens com correlações inferiores a 0,50 (r). 
Numa primeira análise (Tabela 1), verificaram-se os índices de consistência interna e os padrões de correlação existentes entre os itens das seis amostras. Pelo método de análise de componentes principais, com rotação Varimax, forçada a um fator, obtiveram-se as correlações entre os itens (Tabela 1). Todas as amostras verificaram homogeneidade das variáveis [Amostra 1, N = 145, KMO $=0,92\left(\chi^{2}(276)=1951,83 ; p \leq 0,001\right) ;$ Amostra 2 (clínica, indivíduos com diferenças visíveis na face), $N=104, K M O$ $=0,70\left(\chi^{2}(276)=1745,63 ; p \leq 0,001\right)$; Amostra 3 (clínica, indivíduos com diferenças visíveis na face), $N=67, K M O$ $=0,63\left(\chi^{2}(276)=691,66 ; p \leq 0,001 ;\right.$ Amostra 4, $N=214$, $K M O=0,91\left(\chi^{2}(276)=21130,91 ; p \leq 0,001\right)$, Amostra 5, N $=303, K M O=0,87\left(\chi^{2}(276)=2143,85 ; p \leq 0,001\right) \mathrm{e}$ Amostra 6, $N=206, K M O=0,92\left(\chi^{2}(276)=2156,383 ; p \leq\right.$ $0,001)]$. Em todas as amostras, nenhuma das variáveis apresentou valores de assimetria $(S k)$ e curtose $(K u)$ indicadores de violações severas à distribuição normal (| $S k$ $\mid<3$ e $|K u|<10$ ) (Marôco, 2010). Eliminaram-se os itens $1,8,11,12,14,15,17,20,22$ e 24, por apresentarem o maior número de baixas correlações, com exceção do item 19 ("Sentir-se angustiado em encontros sociais").

\section{Tabela 1}

Correlações do Item-Total Corrigido da DAS-24 (Derriford Appearance Scale - reduzida) em todas as amostras ( $\mathrm{N}=1016)$

\begin{tabular}{|c|c|c|c|c|c|c|c|}
\hline Item & Descrição do item & $\begin{array}{l}\text { Amostra } 1 \\
(\alpha=0,94)\end{array}$ & $\begin{array}{l}\text { Amostra } 2 \\
(\alpha=0,89)\end{array}$ & $\begin{array}{l}\text { Amostra } 3 \\
(\alpha=0,79)\end{array}$ & $\begin{array}{l}\text { Amostra } 4 \\
(\alpha=0,90)\end{array}$ & $\begin{array}{c}\text { Amostra } 5 \\
(\alpha=0,87)\end{array}$ & $\begin{array}{l}\text { Amostra } 6 \\
(\alpha=0,91)\end{array}$ \\
\hline 1 & Sentimento de Confiança & 0,66 & $-0,28$ & $-0,16$ & 0,66 & 0,39 & 0,66 \\
\hline 2 & Sentimento de Angústia & 0,76 & 0,63 & 0,57 & 0,70 & 0,60 & 0,71 \\
\hline 3 & Sentir-se irritável em casa & 0,75 & 0,75 & 0,72 & 0,66 & 0,69 & 0,67 \\
\hline 4 & Sentir-se magoado(a) & 0,72 & 0,70 & 0,59 & 0,72 & 0,59 & 0,69 \\
\hline 5 & Autoconsciência afeta ao trabalho & 0,61 & 0,51 & 0,50 & 0,65 & 0,62 & 0,68 \\
\hline 6 & Inquietação na praia & 0,65 & 0,74 & 0,44 & 0,74 & 0,70 & 0,74 \\
\hline 7 & Juízo de valor da aparência & 0,56 & 0,61 & 0,55 & 0,52 & 0,53 & 0,59 \\
\hline 8 & Sentir-se feminino/masculino & 0,31 & $-0,13$ & $-0,20$ & 0,21 & 0,10 & 0,22 \\
\hline 9 & Autoconsciência da aparência & 0,70 & 0,69 & 0,61 & 0,73 & 0,73 & 0,73 \\
\hline 10 & Sentir-se irritado & 0,69 & 0,56 & 0,58 & 0,62 & 0,50 & 0,63 \\
\hline 11 & Utilização de gestos (ocultar) & 0,49 & 0,34 & 0,34 & 0,50 & 0,39 & 0,52 \\
\hline 12 & Evitar vestuários comuns & 0,61 & 0,60 & 0,30 & $-0,03$ & 0,49 & 0,47 \\
\hline 13 & Sentir angústia nos hipermercados & 0,76 & 0,55 & 0,60 & 0,62 & 0,39 & 0,64 \\
\hline 14 & Sentir-se rejeitado & 0,65 & 0,65 & 0,32 & 0,72 & 0,46 & 0,69 \\
\hline 15 & Evitar despir-se frente ao parceiro & 0,64 & 0,44 & 0,31 & 0,18 & 0,34 & 0,18 \\
\hline 16 & Angustiado na prática de desportos & 0,65 & 0,70 & 0,41 & 0,66 & 0,54 & 0,67 \\
\hline 17 & Fechar-se & 0,72 & 0,57 & 0,48 & 0,70 & 0,44 & 0,68 \\
\hline 18 & Preocupar-se por não vestir roupas favoritas & 0,72 & 0,72 & 0,47 & 0,57 & 0,63 & 0,57 \\
\hline 19 & Sentir-se angustiado em encontros sociais & 0,75 & 0,79 & 0,46 & 0,31 & 0,50 & 0,75 \\
\hline 20 & Sentir-se normal & 0,49 & $-0,48$ & $-0,30$ & 0,64 & 0,36 & $-0,26$ \\
\hline 21 & Afetar vida sexual & 0,71 & 0,53 & 0,58 & 0,60 & 0,58 & 0,58 \\
\hline 22 & Evitar sair de casa & 0,68 & 0,39 & 0,01 & 0,57 & 0,33 & 0,59 \\
\hline 23 & Angústia quando comentam a aparência & 0,71 & 0,71 & 0,55 & 0,66 & 0,65 & 0,70 \\
\hline 24 & Evitar cafés/restaurantes & 0,63 & 0,45 & 0,54 & 0,55 & 0,35 & 0,55 \\
\hline
\end{tabular}

Nota. As Amostras 1, 4, 5, 6 constituem amostras não-clínicas e as Amostras 2 e 3 são amostras clínicas. Os itens a sombreado correspondem aos itens eliminados da escala Derriford Appearance Scale - reduzida de 24 itens.

Através da regressão linear múltipla da versão reduzida da DAS-24 a 14 itens e considerando a pontuação total da escala (DAS-14) como variável dependente e os itens que a compõem como variáveis independentes, verificou-se um modelo perfeito para a amostra não clínica $\left(R^{2}=1\right)$ com correlações Item-Total que variaram entre 0,48 a 0,80. Na amostra não-clínica observou-se um modelo quase perfeito $\left(R^{2}=0,94\right)$, com correlações Item-Total que variaram entre 0,48 a 0,67 . Os itens que constituíram a versão reduzida da DAS-24 descreveram-se pelos itens 2 , $3,4,5,6,7,9,10,13,16,18,19,21$ e 23 . Os resultados apresentados seguidamente, concentram-se nas análises dos 14 itens, que apresentaram maiores correlações, quer na amostra clínica, quer na amostra não-clínica. 


\section{Fiabilidade da DAS-14}

A DAS-14 apresentou uma consistência interna elevada para a amostra não clínica $(\alpha$ de Cronbach $=$ 0,91 ), e uma consistência interna moderada a elevada para a amostra clínica ( $\alpha$ de Cronbach $=0,88$ ), estimando boa fiabilidade e precisão da escala.

\section{Validade concorrente da DAS-14 com a ASI-R}

A Tabela 2 mostra que em ambos os grupos de amostras (clínica e não-clínica) se verificaram níveis de intensidade fraca entre a DAS-14 e a dimensão Saliência Motivacional e associações moderadas com a dimensão Saliência Autoavaliativa.

Tabela 2

Correlações de Pearson entre a DAS-14 (Derriford Appearance Scale - reduzida a 14 itens) e as Dimensões da ASI-R (Appearance Schemas Inventory - revista) $(\mathrm{N}=1016)$

\begin{tabular}{|c|c|c|c|c|}
\hline & DAS-14 & $\begin{array}{c}\text { Saliência } \\
\text { Motivacional }\end{array}$ & $\begin{array}{c}\text { Saliência } \\
\text { Autoavaliativa }\end{array}$ & $M(D P)$ \\
\hline \multicolumn{5}{|l|}{ Amostras Não-Clínicas } \\
\hline DAS-14 & - & $0,18^{* *}$ & $0,57^{* *}$ & $21,24(9,72)$ \\
\hline Saliência Motivacional & & - & $0,52 * *$ & $24,25(2,94)$ \\
\hline Saliência Autoavaliativa & & & - & $36,79(5,76)$ \\
\hline \multicolumn{5}{|l|}{ Amostras Clínicas } \\
\hline DAS-14 & - & & & $27,19(10,54)$ \\
\hline Saliência Motivacional & $0,31^{* *}$ & - & & $26,76(5,20)$ \\
\hline Saliência Autoavaliativa & $0,69 * *$ & $0,58^{* *}$ & - & $37,05(8,48)$ \\
\hline
\end{tabular}

Nota. Saliência Motivacional e Saliência Autoavaliativa são dimensões da ASI-R.

${ }^{* *} p<0,01$.

\section{Análise Fatorial Exploratória}

A análise de componentes principais, com rotação Varimax e forçada a um fator, a medida Kaiser-Meyer-Olkin $(K M O)$ que revelou homogeneidade das variáveis e o teste de esfericidade de Bartlett indicaram que as correlações entre os itens eram suficientes para a realização da análise, quer na amostra não-clínica $\left[K M O=0,92 ;\left(\chi^{2}(91)=\right.\right.$ $1339,44 ; p<0,001)]$, quer na amostra clínica $[K M O=$ 0,$\left.84 ;\left(\chi^{2}(91)=1105,05 ; p<0,001\right)\right]$. Quase todos os itens apresentaram correlações moderadas a fortes $(\geq 0,47 ;<$ 0,80), apresentando-se o item 4 da amostra clínica com uma correlação mais fraca em relação aos restantes itens (Tabela 3).

\section{Tabela 3}

Comparação das Correlações do Item-Total Corrigido das DAS-14 numa Amostra Não-Clínica (1) e Amostra Clínica (2)

\begin{tabular}{llcc}
\hline Item & Descrição do Item & Correlação Item-Total (1) & Correlação Item-Total (2) \\
\hline 1 & Sentimento de angústia & 0,76 & 0,65 \\
2 & Sentir-se irritável em casa & 0,75 & 0,78 \\
3 & Sentir-se magoado(a) & 0,67 & 0,63 \\
4 & Autoconsciência afeta o trabalho & 0,66 & 0,47 \\
5 & Inquietação na praia & 0,79 & 0,67 \\
6 & Juízo de valor da aparência & 0,54 & 0,56 \\
7 & Autoconsciência da aparência & 0,81 & 0,72 \\
8 & Sentir-se irritado & 0,65 & 0,65 \\
9 & Sentir angústia nos hipermercados & 0,57 & 0,55 \\
10 & Angustiado na prática de desportos & 0,68 & 0,56 \\
11 & Preocupar-se por não vestir roupas favoritas & 0,73 & 0,60 \\
12 & Sentir-se angustiado em encontros sociais & 0,52 & 0,74 \\
13 & Afetar vida sexual & 0,66 & 0,50 \\
\hline
\end{tabular}




\section{Análise Fatorial Confirmatória}

A estrutura da DAS-14 apresentou índices de qualidade de ajustamento considerados sofríveis na amostra nãoclínica $\left(\chi^{2} / g I=2,68 ; G F I=0,87\right)$ a fracos $(C F I=0,89 ; T L I=$ 0,87; RMSEA = 0,090; $p$ [rmsea $\leq 0,05]<0,001)$, e um mau ajustamento para amostra clínica $\left(\chi^{2} / g l=5,52 ; G F I=0,71\right)$ a ajustamento inaceitável $(C F I=0,67 ; T L I=0,61 ; R M S E A=$ $0,163 ; p[$ rmsea $\leq 0,05]<0,001)$. A regressão e as variâncias de todos os itens em ambos os modelos foram significativas $(p \leq 0,001)$.

Tendo-se verificado um mau ajustamento para o modelo da amostra não-clínica $(G F I=0,87 ; C F I=0,89 ; T L I$ $=0,87)$ e um ajustamento inaceitável para a amostra clinica $(G F I=0,71 ; C F I=0,67 ; T L I=0,61)$, segundo (Marôco, 2014) os índices absolutos não têm grande utilidade quando não comparados com outros modelos, tendo-se assim procedido à análise dos índices de modificação e ao refinamento dos modelos pelas maiores covariâncias apresentadas pelo índice de modificação obtido.

Após reespecificação do modelo da amostra não-clínica (Figura 1) pela correlação dos erros de medida nos itens ( 1 , $2,3,4,5,6,7,8,9,10,11,12$ e 13), o modelo apresentou um ajustamento considerado bom $\left(\chi^{2} / g l=1,17 ; G F I=0,95\right.$; $C F I=0,99 ; T L I=0,98 ; R M S E A=0,028 ; p[$ rmsea $\leq 0,05]=$ $0,92)$. A Figura 2, apresenta a reespecificação do modelo da amostra clínica pela correlação dos erros de medida em todos os itens, tendo-se obtido um ajustamento considerado bom $\left(\chi^{2} / g l=1,36 ; G F I=0,94 ; C F I=0,98 ; T L I\right.$ $=0,96 ;$ RMSEA $=0,047 ; p[$ rmsea $\leq 0,05]=0,56$.

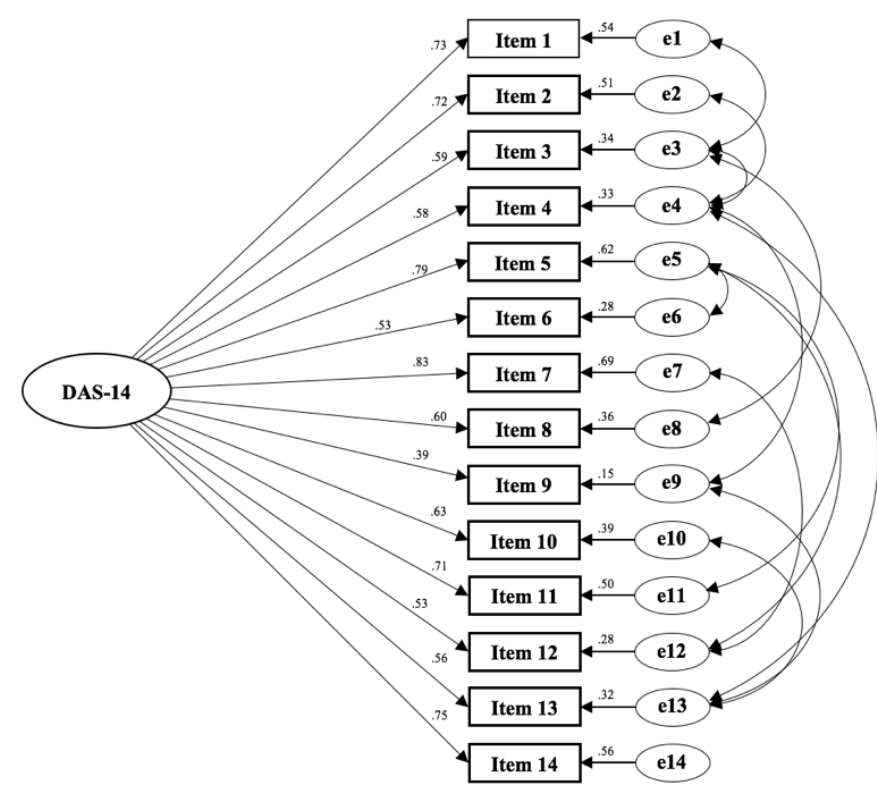

Figura 1. Modelo modificado de análise fatorial confirmatória da amostra não-clínica da DAS-14 $\left(\chi^{2} / g l=\right.$ 1,167; $C F I=0,99 ; G F I=0,95 ; R M S E A=0,028$.

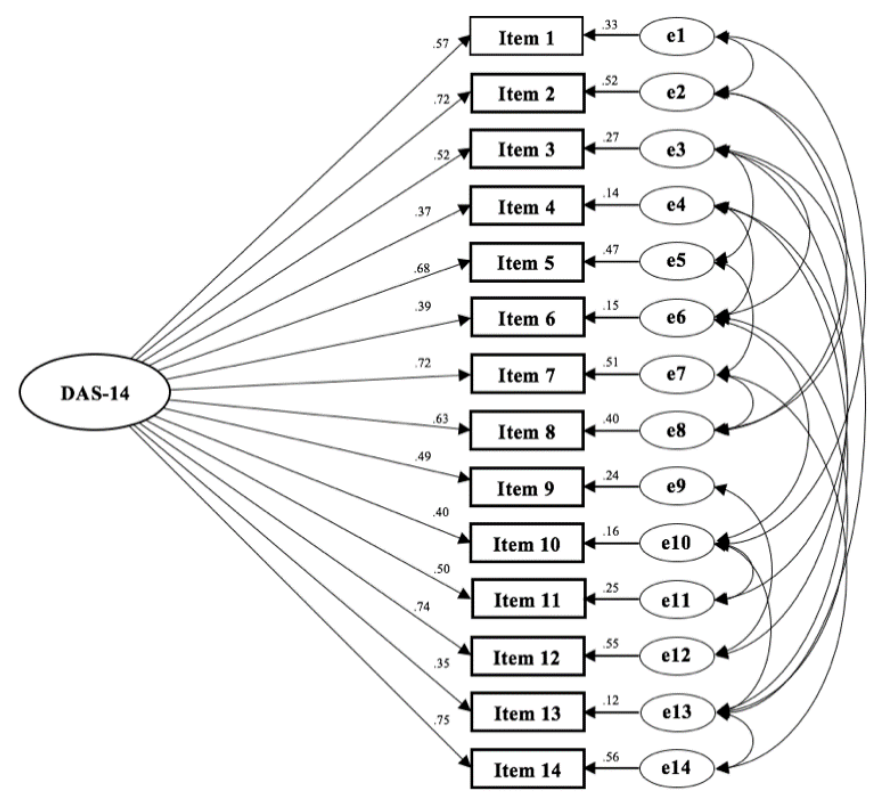

Figura 2. Modelo modificado de análise fatorial confirmatória da amostra clínica da DAS-14 $\left(\chi^{2} / g /=1,368\right.$; $C F I=0,98 ; G F I=0,94 ; R M S E A=0,047)$.

Através dos pesos fatoriais estandardizados do modelo simplificado, calculou-se a fiabilidade compósita que se revelou adequada, quer para a amostra não-clínica $(0,89)$, quer para a amostra clínica $(0,86)$. A VEM, um indicador da validade convergente, revelou-se baixa, quer para a amostra não-clínica $(0,39)$, quer para a amostra clínica $(0,37)$.

\section{DISCUSSÃO}

O impacto psicossocial da aparência é complexo, existindo a necessidade de recorrer a mais do que um instrumento para avaliar possíveis variáveis que influenciam a aparência (Rumsey \& Harcourt, 2005). O feedback da maioria dos participantes dos vários estudos realizados em Portugal (amostras representadas na Tabela 1) que implicam a utilização da DAS-24, quer em contexto clínico, quer em contexto não-clínico, apontam o elevado número de itens como um problema, justificando-se assim o desenvolvimento de uma versão reduzida da Versão Portuguesa da Escala de Avaliação da Aparência Derriford, a DAS-14.

Seguindo as novas linhas de investigação na redução de instrumentos de avaliação que por si já se apresentam como versões reduzidas (Stanton et al., 2002; Tambs \& Røysamb, 2014), procedeu-se à análise de regressão linear múltipla uma vez que o método de preditor dá maior ganho na variância explicada quando inserido em cada etapa (Tambs \& Røysamb, 2014) . Os nossos resultados apontam assim para um modelo perfeito na amostra nãoclínica e um modelo perfeito para a amostra clínica, uma 
vez que nas ciências sociais, um coeficiente de determinação com valores acima de 0,90 são indicadores de um bom ajustamento (Marôco, 2010).

Considerando-se a confiabilidade uma propriedade muito importante num instrumento (Widaman, Little, Preacher, \& Sawalani, 2011), a versão reduzida das DAS24 apresenta-se como uma medida de precisão, determinando a pontuação de um indivíduo na escala. Os 14 itens da Derriford Appearance Scale na amostra nãoclínica apresenta um alfa de Cronbach de 0,91, considerando-se fiabilidade elevada, tendo-se obtido uma fiabilidade moderada a elevada para a amostra clínica ( $\alpha$ de Cronbach $=0,88$ ) (Marôco \& Garcia-Marques, 2006). $\mathrm{Na}$ análise fatorial exploratória dos 14 itens que constituem a escala, verifica-se homogeneidade das variáveis, quer na amostra não-clínica $(K M O=0,92)$, quer na amostra clínica $(K M O=0,84)$ considerados de bom a elevada (Marôco, 2010), apresentando correlações moderadas em quase todos os itens $(0,50 \leq r \leq 0,80)$, à exceção do item 4 na amostra clínica $(r=0,47)$. Uma vez que esta amostra foi recolhida em contexto hospitalar (internamento), os indivíduos podem ter respondido de forma aleatória ao item 4 - "No momento presente, o desconforto/constrangimento que sinto em relação à minha aparência tem um efeito negativo no meu trabalho" - , justificando-se assim a baixa correlação, devido aos indivíduos ainda não terem estabelecido qualquer relação interpessoal no meio laboral.

Através da análise fatorial confirmatória, o primeiro modelo de equação estrutural testado em ambas as amostras, apresenta índices de qualidade do ajustamento sofrível, pelo que, através da modificação dos erros estimados pela maior covariância, obtiveram-se melhores níveis de qualidade de ajustamento.

Atendendo às limitações deste estudo, especificamente à impossibilidade de avaliar se os participantes da amostra não-clínica apresentavam algum tipo de diferença visível na face que pudesse comprometer os resultados; a falta de estudos que reportam resultados da DAS-24 e ao número reduzido de participantes na amostra clínica, a DAS-14 apresenta-se como uma escala reduzida da Derriford Appearance Scale para a população portuguesa, com boas propriedades psicométricas, quer na população geral, quer na população clínica (apresentam diferenças visíveis).

O desenvolvimento da versão reduzida da Escala de Avaliação da Aparência de Derriford (DAS-14) permite a sua aplicação em contexto clínico e respetiva investigação, uma vez que a sua administração requer menos tempo, facilitando a integração de outros instrumentos de avaliação.
Conflito de interesses | Conflict of interest: Os autores declaram que não existem quaisquer conflitos de interesses. No entanto, a autorização para uso da versão reduzida da Escala de Avaliação da Aparência de Derriford (DAS-14), deve ser solicitada aos autores da versão original da DAS-24 (tim.moss@uwe.ac.uk) e versão portuguesa (hmoreira@fpce.uc.pt)| The authors declare that there are no conflicts of interest. However, authorization to use the reduced version of the Derriford Appearance Assessment Scale (DAS-14) should be requested from the authors of the original version of DAS-24 (tim.moss@uwe.ac.uk) and the Portuguese version (hmoreira@fpce.uc.pt).

Fontes de financiamento | Funding sources: Nenhuma | None.

Agradecimentos | Acknowledgements: À Professora Doutora Helena Moreira na revisão parcial do presente trabalho | To Professor Helena Moreira in the partial revision of the present work.

\section{REFERÊNCIAS}

Alves, D. (2016). Transplante da face: Impacto na análise da expressão facial da emoção [Transplantation of the face: Impact on the analysis of facial expression of emotion]. Santo Tirso: Idioteque.

Carr, T., Harris, D., \& James, C. (2000). The Derriford Appearance Scale (DAS-59): A new scale to measure individual responses to living with problems of appearance. British Journal of Health Psychology, 5(2), 201-215. https://doi.org/10.1348/135910700168865

Carr, T., Moss, T., \& Harris, D. (2005). The DAS24: A short form of the Derriford Appearance Scale DAS59 to measure individual responses to living with problems of appearance. British Journal of Health Psychology, 10(2), 285-298. https://doi.org/10.1348/135910705X27613

Cash, T., Melnyk, S., \& Hrabosky, J. (2004). The assessment of body image investment: An extensive revision of the appearance schemas inventory. The International Journal of Eating Disorders, 35(3), 305-316. https://doi.org/ 10.1002/eat.10264

Chaer, G., Diniz, R., \& Ribeiro, E. (2011). A técnica do questionário na pesquisa educacional [The questionnaire technique in educational research]. Evidência, 7(7), 251-266. Retrived from http://www.educadores.diaadia.pr.gov.br/arquivos/File/maio20 13/sociologia_artigos/pesqusia_social.pdf

Harris, D., \& Carr, A. (2001a). Prevalence of concern about physical appearance in the general population. British Journal of Plastic Surgery, 54(3), 223-226. https://doi.org/10.1054/bjps.2001.3550

Harris, D., \& Carr, A. (2001b). The Derriford Appearance Scale (DAS59): A new psychometric scale for the evaluation of patients with disfigurements and aesthetic problems of appearance. British Journal of Plastic Surgery, 54(3), 216-222. https://doi.org/10.1054/bjps.2001.3559

Leeuw, E., Hox, J., \& Dillman, D. (2008). International handbook of survey methodology. London: Lawrence Erlbaum Associates.

Marôco, J. (2010). Análise estatística com o PASW Statistics (ex-SPSS) [Statistical analysis with PASW Statistics (ex-SPSS)]. Lisboa: ReportNumber.

Marôco, J. (2014). Análise de equações estruturais: Fundamentos teóricos, software \& aplicações [Theoretical fundamentals, software \& applications] (2nd ed.). Lisboa: ReportNumber.

Marôco, J., \& Garcia-Marques, T. (2006). Qual a fiabilidade do alfa de Cronbach? Questões antigas e soluções modernas? [How reliable is Cronbach's alpha? Old issues and modern solutions?]. Laboratório de Psicologia, 4(1), 65-90. Retrived from http://publicacoes.ispa.pt/index.php/lp/article/viewFile/763/706

Mendes, J., \& Figueiras, M. (2013). Acquired facial disfigurement: A brief narrative review. Psicologia, Saúde \& Doenças, 14(3), 484501. https://doi.org/10.15309/13psd140309

Mendes, J., Figueiras, M., \& Moss, T. (2016). Influence of personality traits in self-evaluative salience, motivational salience and self- 
consciousness of appearance. Psychology, Community \& Health 5(3), 187-197. https://doi.org/10.5964/pch.v5i2.168

Mendes, J., Figueiras, M., Moreira, H., \& Moss, T. (2016). Análise fatorial da versão portuguesa da Escala de Avaliação da Aparência de Derriford (DAS-24) [Factorial validity of the Derriford Appearance Scale (DAS-24) Portuguese Version]. Psychology, Community \& Health, 5(3), 31-43. https://doi.org/10.5964/pch.v5i1.128

Mendes, J., Rego, R., Figueiras, M., \& Moss, T. (2018). Facial disfigurement - a multidimensional approach: Theory and models. Manuscript submitted for publication.

Mendes, J., Rego, R., Figueiras, M., Moss, T., \& Alcaidinho, D. (2018). Psychological adjustment to acquired facial disfigurement: Personality characteristics, self-concept and satisfaction with social support - longitudinal study. Manuscript submitted for publication.

Nazaré, B., Moreira, H., \& Canavarro, M. C. (2010). Uma perspectiva cognitivo-comportamental sobre o investimento esquemático na aparência: Estudos psicométricos do Inventário de Esquemas sobre a Aparência (ASI-R) [A cognitive-behavioral perspective on schematic investment in appearance: Psychometric studies of the Appearance Schemas Inventory (ASI-R)]. Laboratório de Psicologia, 8(1), 21-36. Retrived from http://repositorio.ispa.pt/bitstream/10400.12/3424/1/LP_8_21-36.pdf

Ribeiro, J. (2010). Metodologia de investigação em psicologia e saúde
[Methodology of research in psychology and health] (3rd ed.). Lisboa: Livpsic.

Rosser, B., Moss, T., \& Rumsey, N. (2010). Attentional and interpretative biases in appearance concern: An investigation of biases in appearance-related information processing. Body Image, 7(3), 251-254. https://doi.org/10.1016/j.bodyim.2010.02.007

Rumsey, N., \& Harcourt, D. (2005). The psychology of appearance. England: Open University Press.

Stanton, J., Sinar, E., Balzer, W., \& Smith, P. (2002). Issues and strategies for reducing the length of self-report scales. Personnel Psychology, 55, 167-194. https://doi.org/10.1111/j.1744-6570.2002.tb00108.x

Tambs, K., \& Røysamb, E. (2014). Selection of questions to shortform versions of original psychometric instruments in MoBa. Norsk Epidemiologi, 24(1-2), 195-201. https://doi.org/10.5324/nje.v24i1-2.1822

Widaman, K., Little, T., Preacher, K., \& Sawalani, G. (2011). On creating and using short forms of scales in secondary research. In B. Donnellen \& K. Trzesniewski (Eds.), Archival data analysis (pp. 39-61). Washington, DC: American Psychological Association.

Ziegler, M., Kemper, C., \& Kruyen, P. (2014). Short scales - five misunderstandings and ways to overcome them. Journal of Individual Differences, 35(4), 185-189. https://doi.org/10.1027/1614-0001/a000148 